\title{
Die Reaktionsweise von Investoren auf affektive Managementstatements
}

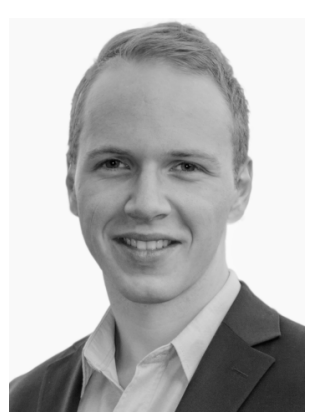

Joachim Lang

Mittels fiktiver affektiver Managementstatements wurde untersucht, ob die Erwartungen und Einschätzungen verschiedener Anlegertypen bei gleicher Zahlen- und Faktenlage durch den Einsatz von linguistischen Treatments variieren. Die Resultate suggerieren, dass unerfahrene Privatanleger einen signifikant höheren (tieferen) Aktienreturn prognostizieren, wenn sie positiv (negativ) formulierte Managementstatements lesen. Weiter zeigen die Ergebnisse auf, dass die unerfahrenen Privatanleger der Meinung sind, dass die Gesamtheit der Investoren einen signifikant höheren (tieferen) Aktienreturn prognostiziert, wenn positiv (negativ) formulierte Managementstatements gelesen wurden. Es lässt sich schlussfolgern, dass die Art der Formulierung in den Augen dieses Investorentyps, bewusst und/oder unbewusst wirkend, relevante Informationen enthält. Aus einer praktischen Sicht würde dies bedeuten, dass seitens des Managements systematisch auf die Erwartungen und Einschätzungen unerfahrener Privatanleger Einfluss genommen werden kann. Bei den professionellen Anlegern und den erfahrenen Privatanlegern konnten diese Wirkungszusammenhänge nicht festgestellt werden.

Using fictitious affective management statements, it was examined whether the expectations and evaluations of different types of investors vary due to the use of linguistic treatments while numbers and facts remain unchanged. The results suggest that unexperienced private investors predict a significantly higher (lower) stock return if they read positively (negatively) formulated management statements. Further, the results indicate that unexperienced private investors tend to expect that the aggregate of investors predicts a significantly higher (lower) stock return if positively (negatively) formulated management statements are read. It can be concluded that in the eyes of this type of investor, consciously and/or unconsciously influencing, the wording contains substantial information. From a practical point of view, this would imply that the management could systematically influence the expectations and evaluations of unexperienced private investors. These interdependencies could not be identified in the case of professional investors and experienced private investors.

Managementstatements, Sentiment, Tone, Erwartungen, Anlegertypen, Aktienreturn, Volatilität

Management Statements, Sentiment, Tone, Expectations, Investor Types, Stock Return, Volatility 


\section{Einleitung}

Täglich sehen sich Investoren mit einer Informationsflut konfrontiert. Zu Beginn der Informationsverarbeitungskette treffen Informationen jeglicher Art über einen oder mehrere Kanäle auf die Investoren. Bei den Investoren angekommen, werden die Informationen von ihnen verarbeitet und anhand des Resultates dieses Verarbeitungsprozesses treffen die Investoren Entscheidungen. Im eigentlichen Verarbeitungsprozess beeinflussen die Informationen qualitativer und quantitativer Natur die Erwartungen und Einschätzungen der Investoren.

In dieser Arbeit wurde ein experimenteller Ansatz zur Untersuchung der Wirkung von qualitativer Information auf Investoren gewählt. Mittels fiktiver affektiver Testmanagementstatements wurde untersucht, ob die Erwartungen und Einschätzungen der Investoren bei gleicher Zahlen- und Faktenlage durch den Einsatz von linguistischen Treatments variieren. Anders formuliert wird in dieser Untersuchung der Frage nachgegangen, ob die sprachliche Gestaltung von Managementstatements ergänzend zu den gelieferten Zahlen und Fakten die Erwartungen und Einschätzungen der Investoren beeinflusst.

Variieren die Erwartungen und Einschätzungen mit der Art der Formulierung, enthält diese in den Augen der Investoren, bewusst und/oder unbewusst wirkend, relevante Informationen. Erzeugen diese Informationen bewusst und/oder unbewusst Erwartungs- und Einschätzungsänderungen, dann beeinflussen sie die von den Investoren getroffenen Entscheidungen. Die getroffenen Entscheidungen haben Handelsaktivität zur Folge, welche sich in den Marktpreisen niederschlägt. Demnach würde die Art der Formulierung über den beschriebenen Wirkungskanal auf die Handelsaktivität und auf die Marktpreisbewegungen Einfluss nehmen.

Nahezu alle durchgeführten Studien zum Thema der textbasierten Sentiment-Analyse sind Event-Studien. Mittels der im Rahmen dieser Arbeit durchgeführten Umfrage wurde eine neuartige Informationsquelle für die Analyse textbasierten Sentiments generiert. In bisherigen Forschungsbeiträgen hat man sich darauf konzentriert, die Wirkung der Worte der linguistischen Kategorien Positiv und insbesondere Negativ zu untersuchen. Als Erweiterung wurde in dieser Umfrage das Ziel verfolgt, die Wirkung der Worte der linguistischen Kategorien Positiv, Negativ, Unsicher und Rechtlich/Strittig zu untersuchen.

In der Umfrage wurden die Teilnehmer gebeten, ihnen vorgelegte fiktive affektive Managementstatements zu lesen und jeweils vier identische Fragen dazu zu beantworten. Aus den abgegebenen Schätzungen konnten Aktienreturn-Prognosen, Volatilitätsprognosen, Durchschnittsreturn-Prognosen und die gewählte Handlungsoption (Buy/Hold/Sell) abgeleitet werden. Mittels Fragen zur Person wurden die Umfrageteilnehmer in die drei Investorentypen professionelle Anleger, erfahrene Privatanleger und unerfahrene Privatanleger unterteilt. Das gewählte Umfragedesign hatte den Vorteil, dass jedem Umfrageteilnehmer zu jedem der fiktiven Unternehmen nur eine Version der fiktiven Managementstatements vorgelegt wurde. Dieses Konstrukt der implementierten Kontrollgruppen und eingebaute Randomisierungen stellten die Voraussetzungen dar, um kausale Zusammenhänge eruieren zu können.

Die Resultate zu den Aktienreturn-Prognosen suggerierten, dass unerfahrene Privatanleger einen signifikant höheren (tieferen) Aktienreturn prognostizieren, wenn sie positiv (negativ) formulierte Managementstatements lesen. Bei den professionellen Anlegern und den erfahrenen Privatanlegern konnte dieser Wirkungszusammenhang nicht festgestellt werden. Dies würde bedeuten, dass mit der Art der Formulierung im Fall von positiver bzw. 
negativer Einfärbung systematisch Einfluss auf die Erwartungen und Einschätzungen der unerfahrenen Privatanleger genommen werden kann.

Die Ergebnisse zu den Durchschnittsreturn-Prognosen suggerierten, dass die unerfahrenen Privatanleger der Meinung sind, dass die Gesamtheit der Investoren einen signifikant höheren (tieferen) Aktienreturn prognostiziert, wenn positiv (negativ) formulierte Managementstatements gelesen wurden. Bei den professionellen Anlegern und den erfahrenen Privatanlegern war es erneut so, dass der bei den unerfahrenen Privatanlegern existierende Wirkungszusammenhang für sie nicht festgestellt werden konnte.

Bei allen Investorentypen war es zutreffend, dass auf der Individuum-Ebene erwartet wurde, dass die Gesamtheit der Investoren ihre Erwartungen zu den Aktienreturn-Prognosen bezüglich positiv bzw. negativ formulierter Managementstatements auf die gleiche Weise bildeten wie sie selbst. Während die professionellen Anleger und die erfahrenen Privatanleger davon ausgingen, dass die Tonalität ("Tone") in Managementstatements sowohl auf sie als auch auf die unerfahrenen Privatanleger keine signifikante Wirkung erzielt, gingen die unerfahrenen Privatanleger davon aus, dass der Tone in Managementstatements sowohl auf sie als auch auf die professionellen Anleger und die erfahrenen Privatanleger eine signifikante Wirkung erzielt.

Wird in diesem Beitrag von Reaktionsweise gesprochen, bezieht sich dies auf die Schnittstelle zwischen Informationseingang und der Verarbeitung der Information. Die Reaktionsweise ist die durch den Informationseingang bedingte Erwartungs- bzw. Einschätzungsanpassung. In Abgrenzung dazu sind die aus den Erwartungs- und Einschätzungsanpassungen abgeleiteten Handelsentscheidungen nicht Teil der Reaktionsweise. Investoren lassen sich anhand ihrer Anlegertypologie differenzieren. Bei der Auswertung der Umfrageergebnisse wurde zwischen den drei Anlegertypen professionelle Anleger, erfahrene Privatanleger und unerfahrene Privatanleger unterschieden. Die Beobachtungen der beiden Anlegertypen institutionelle Investoren und Finanzberater wurden zur Gruppe der professionellen Anleger zusammengefasst. Umfrageteilnehmer wurden der Gruppe erfahrene (unerfahrene) Privatanleger zugeordnet, wenn sie bei der Frage nach der vorhandenen Investitionserfahrung einen Wert von drei und mehr (von weniger als drei) angegeben hatten, wobei der minimale Wert von eins keiner und der maximale Wert von fünf sehr viel Investitionserfahrung gleichkam.

Wird in diesem Beitrag von einem affektiven Statement gesprochen, ist damit ein gefühlsbetontes Statement bzw. ein Statment mit enthaltenen Konnotationen gemeint. In den konstruierten Testmanagementstatements wurde durch das Einbauen von linguistischen Treatments darauf abgezielt, zu untersuchen, ob die auf die Gefühlsbetonung wirkende Einfärbung Erwartungs- und Einschätzungsvariationen bei den Investoren zur Folge hat. Beim Begriff Managementstatement wird zwischen Managementstatements und Testmanagementstatements differenziert. Wird von Managementstatements gesprochen, ist die Management Discussion and Analysis (MD\&A) gemeint. Bei den Testmanagementstatements handelt es sich um die konstruierten Managementstatements fiktiver Natur.

\section{Literatur}

Aus Sicht der klassischen Kapitalmarkttheorie entsprechen Wertpapierpreise im Gleichgewicht den rationalen Erwartungen der diskontierten zukünftigen erwarteten Cashflows. Der Querschnitt der erwarteten Returns wird durch den Querschnitt der systematischen Risiken determiniert. Aussergewöhnliche Ereignisse wie der Schwarze Donnerstag im Ok- 
tober 1929 oder der Schwarze Montag im Oktober 1987, die zu starken Wertpapierpreisänderungen geführt haben, sind durch die klassische Kapitalmarkttheorie nicht erklärbar. Preisbildungsmodelle des Forschungszweigs der Behavioral Finance versuchen, sich der Realität des Marktgeschehens anzunähern. Investoren-Sentiment hat in der klassischen Kapitalmarkttheorie keine Existenzberechtigung. (Baker \& Wurgler, 2006, 2007)

Der Begriff Sentiment ist in diesem Beitrag von zentraler Bedeutung. In der Vergangenheit wurden zwei Typen von Sentiments untersucht. Beim Investoren-Sentiment handelt es sich um einen zusammenfassenden Begriff für Zukunftsüberzeugungen zu Investitionsrisiken und Cashflows, die durch fundamentale Daten nicht begründbar sind. Beim zweiten Sentimenttyp, dem textbasierten Sentiment, handelt es sich um affektive linguistische Einfärbungen. Ist die Rede von positiver oder negativer Einfärbung, wird in diesem Zusammenhang oftmals von Tone gesprochen. Die Verbindung und die kausalen Beziehungen von Investoren-Sentiment und textbasiertem Sentiment sind ausgesprochen komplex. Während das Investoren-Sentiment eher subjektive Überzeugungen der Investoren einfängt, tendiert das textbasierte Sentiment dazu, eine objektivere Betrachtung der Gegebenheiten auf Märkten und in Unternehmen wiederzugeben. (Baker \& Wurgler, 2007; De Long, Shleifer, Summers, \& Waldmann, 1990; Kearney \& Liu, 2014; Shleifer \& Vishny, 1997)

De Long et al. (1990) unterscheiden zwischen Investoren mit rationalen Erwartungen und Noise Tradern. In Anlehnung an Black (1986) handelt es sich bei Noise um nicht-fundamentale Volatilität. Risiko fundamentaler Art und Risiko nicht-fundamentaler Art begrenzen das Potenzial von Arbitrage. Durch die Potenzialbegrenzung der Arbitrage sind Marktpreise denkbar, die von ihren fundamentalen Werten abweichen. Noise Trading kann zu grossen Abweichungen zwischen Marktpreisen und ihren fundamentalen Werten führen. (De Long et al., 1990; Shleifer \& Vishny, 1997)

Baker and Wurgler (2006) postulieren, dass Marktpreise von ihren fundamentalen Werten abweichen und dass dies einerseits durch die Potenzialbegrenzungen von Arbitrage und andererseits durch Sentiment-basierte Nachfrageschocks zustande kommt. In einer jüngeren Studie konstatieren Baker \& Wurgler, dass es nicht länger eine Frage sei, ob Investoren-Sentiment die Preise beeinflusst, sondern wie man Investoren-Sentiment messen sollte und wie man die durch Investoren-Sentiment erzeugten Effekte quantifizieren kann. (Baker \& Wurgler, 2006, 2007)

Textbasierte Sentiment-Analysen stellen im Forschungszweig der Behavioral Finance einen an Wichtigkeit zunehmenden Ansatz dar. Von Interesse sind die kausalen Beziehungen von Investoren-Sentiment und textbasiertem Sentiment. Beeinflusst textbasiertes Sentiment das Investoren-Sentiment, dann ist eine Inklusion von qualitativen Informationen der textbasierten Sentiment-Analysen in Asset-Pricing-Modelle als sinnvolle Erweiterung anzusehen. (Kearney \& Liu, 2014)

Die drei wichtigsten Informationsquellen für textbasierte Sentiment-Analysen sind öffentliche Berichterstattungen von Unternehmen, mediale Berichterstattung und aus dem Internet stammende Inhalte wie bspw. Mitteilungen aus den sozialen Medien. Die beiden verbreitetsten methodischen Herangehensweisen zur Bestimmung des textbasierten Sentiments sind Machine Learning und der Wörterbuchansatz. Bei letzterer Methodik werden die in Texten enthaltenen Wörter mit einer Wortliste abgeglichen, die linguistische Kategorien enthält. Liegt eine Übereinstimmung vor, wird das im Text enthaltene Wort entsprechend der Kategorieübereinstimmung klassifiziert. Von besonderer Relevanz ist bei diesem 
Ansatz die Wahl der Wortliste und die gewählte Gewichtung der klassifizierten Wörter. Bei der Einarbeitung der linguistischen Treatments in die fiktiven affektiven Managementstatements, die den Umfrageteilnehmern vorgelegt wurden, sind für die Klassifizierung der relevanten Textstellen die für finanzielle Kontexte geeigneten Wortlisten von Loughran and Mcdonald (2011) verwendet worden. Wird zur Bestimmung des textbasierten Sentiments der Wörterbuchansatz gewählt, ist die geläufigste Messmethode die Anteilsermittlung. Zur Anteilsermittlung dividiert man die Anzahl einer linguistischen Kategorie zugehörigen klassifizierten Wörter durch die Gesamtwortanzahl eines Textes. Ist man bspw. an der Negativität eines Textes interessiert, enthält man den gesuchten Anteilswert, indem man die Anzahl Wörter dieses Textes, die als negativ klassifiziert wurden, durch die Gesamtwortanzahl des Textes dividiert. (Kearney \& Liu, 2014)

Die Studienresultate von Tetlock (2007) suggerieren im Sinne der Sentiment-Theorie, dass ein hoher Level von Medienpessimismus signifikant fallende Marktpreise mit darauffolgender Reversion zu den fundamentalen Werten vorhersagen kann. Kurzfristig könnten damit demnach fallende Marktpreise und längerfristig steigende Marktpreise vorhergesagt werden. Gemäss den gefundenen Resultaten reversieren die Marktpreise innerhalb einer Woche. Implementiert man die gefundene Vorhersagekraft des Medienpessimismus in eine hypothetisch kostenfreie Handelsstrategie, resultiert im Vergleich zur Marktbenchmark ein Überschussreturn von 4.4 Basispunkten pro Tag, was einer jährlichen Überschussrendite von $7.3 \%$ gleichkommt. Adjustiert man jedoch die Überschussreturns um realistische durch den Handel verursachte Kosten, ist es fragwürdig, ob diese weiterhin signifikant positiv verschieden von Null sind.

Die Resultate der Studie von Tetlock et al. (2008) deuten darauf hin, dass negative Worte in unternehmensspezifischer medialer Berichterstattung tiefe Unternehmensgewinne voraussagen können und keine redundanten Informationen darstellen. Es scheint sich dabei um relevante Informationen zu den Unternehmenskennzahlen zu handeln, die nur schwer zu quantifizieren sind. Inkludiert man realistische, durch den Handel verursachte Kosten und Kapitalgewinnsteuern in mögliche Daytrading-Strategien, ist es fragwürdig, ob diese profitabel sind. Weiter stellen die Autoren fest, dass negative Worte in unternehmensspezifischen Textkorpussen, in welchen es um Unternehmenskennzahlen geht, Unternehmensgewinne und Unternehmensreturns deutlich besser als negative Worte in unternehmensspezifischen Textkorpussen, in welchen es nicht um Unternehmenskennzahlen geht, voraussagen können. Investoren scheinen zwischen Berichterstattung zu Unternehmensgewinnen und Berichterstattung zu anderen Themen zu unterscheiden.

In der Studie von $\mathrm{Li}$ (2010) wurden die zukunftsgerichteten Statements (FLS) der MD\&A untersucht. Die Resultate suggerieren, dass der durchschnittliche Tone in zukunftsgerichteten Statements positiv mit der Liquidität und den zukünftigen Gewinnen des Unternehmens korreliert ist. Die gemessenen Tone-Werte haben jedoch keinen signifikanten Erklärungsgehalt für zukünftige Unternehmensperformance.

In Bosman et al. (2015) stellen die Informationsquelle für textbasiertes Sentiment die Resultate einer durchgeführten Umfrage dar. Studentinnen und Studenten wurde ein Ausschnitt eines News-Artikels und ein Chart mit vergangener Aktienpreisentwicklung zu einem Unternehmen als Informationsset vorgelegt. Sie wurden gebeten, anhand des vorgelegten Informationssets Aussagen gemäss ihren Einschätzungen zum Unternehmen zu treffen. Es wurde bspw. um eine Schätzung des Aktienpreises am Ende der nächsten Handelsperiode gebeten. Durch Modifikationswörter wurden Textstellen gezielt akzentuiert bzw. 
abgeschwächt. Mit der Umfrage wurde das Ziel verfolgt, die Wirkung von News-Tone auf die Aktienpreiserwartungen und Überzeugungen einzelner Investoren zu untersuchen. Die Resultate zeigen, dass der News-Tone signifikant die Aktienpreisentwicklungsschätzung beeinflusst. Die geschätzten Werte bei negativ (positiv) eingefärbten Informationssets sind signifikant tiefer (höher) als bei positiv (negativ) eingefärbten Informationssets. Bei den Erwartungen zur zukünftigen Volatilität konnten keine signifikanten, durch die Tone-Wirkung erzeugten Unterschiede ausgemacht werden. Weiter deuten die Resultate darauf hin, dass die Wahrscheinlichkeiten für Kauf- und Verkaufsentscheidungen von Unternehmensanteilen durch den News-Tone beeinflusst werden. (Bosman et al., 2015)

Nahezu alle durchgeführten Studien zum Thema der textbasierten Sentiment-Analyse sind Event-Studien. Mittels der im Rahmen dieser Arbeit durchgeführten Umfrage wurde eine neuartige Informationsquelle für die Analyse textbasierten Sentiments generiert. In bisherigen Forschungsbeiträgen hat man sich darauf konzentriert, die Wirkung der Worte der linguistischen Kategorien Positiv und insbesondere Negativ zu untersuchen. In dieser Arbeit wurde als Erweiterung das Ziel verfolgt, die Wirkung der Worte der linguistischen Kategorien Positiv, Negativ, Unsicher und Rechtlich/Strittig zu untersuchen.

\section{Methodik}

Im ersten Teil dieses Kapitels wird auf die Struktur der Umfrage eingegangen. Im zweiten Teil wird beschrieben, welche inhaltlichen Zusammenhänge bei den Ergebnissen erwartet wurden und die daraus abgeleiteten Hypothesen werden gezeigt. Im abschliessenden dritten Teil werden die erhobenen Daten erläutert.

\subsection{Struktur der Umfrage}

Die Umfrageteilnehmer wurden gebeten, ihnen vorgelegte fiktive affektive Managementstatements zu lesen und Fragen dazu zu beantworten. In die fiktiven affektiven Managementstatements wurden linguistische Treatments eingearbeitet. Die durch die Umfrageteilnehmer gegebenen Antworten erlaubten es, zu untersuchen, wie verschiedene Investorentypen auf textbasiertes Sentiment reagieren bzw. inwiefern textbasiertes Sentiment Einschätzungen und Erwartungen beeinflusst.

\subsubsection{Umfrageverlauf}

Hatte ein Umfrageteilnehmer die Umfrage geöffnet, wurde er randomisiert mit gleicher Wahrscheinlichkeit einem von drei Strängen zugewiesen. Ohne zu wissen, in welchem Strang er sich befand, sah er ein fiktives Managementstatement und die dazugehörigen Fragen vor sich. Auf dieser Stufe wurde ebenfalls mit gleicher Wahrscheinlichkeit randomisiert. Nachdem die sechs Statements und die dazugehörigen Fragen in zufälliger Reihenfolge nacheinander erschienen waren, gelangte der Umfrageteilnehmer zu den Fragen zur Person.

Für sechs verschiedene fiktive Unternehmen wurden jeweils drei variierende fiktive Managementstatements verfasst. Dieses Umfrageverlaufsdesign hatte den Vorteil, dass jedem Umfrageteilnehmer zu jedem Unternehmen jeweils nur eine Version der fiktiven Managementstatements vorgelegt wurde. Die Randomisierung auf der Strangzuordnungsebene und die Randomisierung der vorgelegten fiktiven Managementstatements zielten darauf ab, Störfaktoren (Confounding Factors) auf ein Minimum zu reduzieren. Für jedes der fik- 
tiven Unternehmen wurde eines der fiktiven Statements zur Kontrolle als neutrales Referenzstatement formuliert. Die Randomisierung und das eingebaute Konstrukt der Kontrollgruppen stellten die Voraussetzungen dar, um die Herausarbeitung bzw. die Messung von kausalen Zusammenhängen bewerkstelligen zu können. (Stock \& Watson, 2011)

\subsubsection{Konstruktion der fiktiven affektiven Managementstatements}

In einem ersten Schritt wurden sechs verschiedene Managementstatements fiktiver Natur verfasst. In ihnen wurden szenische Unternehmenskontexte wie bspw. Gewinnveränderungen, Umsatzveränderungen oder veränderte Marktbedingungen eingebaut. Auf Informationen wie Unternehmensnamen, Personennamen oder zeitlicher Kontext wurde bewusst verzichtet, um unkontrollierbare Effekte auf ein Minimum zu beschränken. Die sechs Statements wurden als neutrale Referenz definiert und stellten den Ausgangspunkt für das weitere Vorgehen dar. In einem zweiten Schritt wurden zu jedem der Referenzstatements zwei Treatment-Statements verfasst. Zu den Unternehmen 1-3 wurde jeweils ein positiv konnotiertes und ein negativ konnotiertes Treatment-Statement verfasst. Zu den Unternehmen 4-6 wurde jeweils ein unsicher konnotiertes und ein rechtlich/strittig konnotiertes Treatment-Statement verfasst. Dabei wurde versucht, den durch die Zahlen- und Faktenlage definierten Inhalt durch die nötigen Veränderungen bei den jeweiligen Treatments nicht zu verzerren. Gleichzeitig musste sichergestellt werden, dass die Konnotationen nicht auf einer subjektiven Ebene verbleiben. Deshalb wurde systematisch mit möglichst objektiven Mitteln wie bspw. Adjektivsteigerungen oder Antonymen gearbeitet, weil diese unabhängig vom eigenen Sprachempfinden sind. Hierbei handelt es sich um konventionalisierte Formen. Dadurch war es möglich, die Einordnung der Treatments zu objektivieren.

\subsubsection{Der Fragebogen}

$\mathrm{Zu}$ jedem der sechs vorgelegten Statements wurden jedem Umfrageteilnehmer jeweils vier identische Fragen gestellt.

Als erstes wurde der Umfrageteilnehmer um eine Schätzung der prozentualen Aktienpreisveränderung bis in einem Monat gebeten. Mittels des hier angegebenen Wertes wurde die Aktienreturn-Erwartung $\left(r_{i j}^{e x p}\right)$ eines Umfrageteilnehmers $\mathrm{j}$ für das Unternehmen i mit der nachfolgenden Formel ermittelt.

$r_{i j}^{\text {exp }}=\ln \left(\frac{\text { Preisveränderung }}{i j}+1\right)$

Als zweites wurde der Umfrageteilnehmer $\mathrm{j}$ gebeten, zu schätzen, wie sich der Aktienpreis des Unternehmens i prozentual bis in einem Monat im besten Fall (= Upper Bound) und wie sich der Aktienpreis prozentual bis in einem Monat im schlechtesten Fall (= Lower Bound) verändert. Mittels der hier angegebenen Werte wurde die erwartete Volatilität $\left(\sigma_{i j}^{\exp }\right.$ ) des Umfrageteilnehmers j für das Unternehmen i mit der untenstehenden Formel ermittelt. Gemäss Bosman et al. (2015) kann mittels untenstehender Formel die erwartete Volatilität zum Konfidenzniveau von $90 \%$ geschätzt werden.

$\sigma_{i j}^{\text {exp }}=\frac{\left[\ln \left(\frac{\text { upper }_{i j}}{100}+1\right)-\ln \left(\frac{\text { Lower }_{i j}}{100}+1\right)\right]}{2.65}$ 
Als drittes wurde der Umfrageteilnehmer j gebeten, zu schätzen, welcher Wert bei der Frage nach der erwarteten prozentualen Aktienpreisentwicklung durchschnittlich durch den gesamten Pool der N Umfrageteilnehmer angegeben wurde $\left(\frac{1}{N} \sum_{j}^{N} r_{i j}^{\text {exp }}\right)$. Mittels des hier angegebenen Wertes wurde die durchschnittliche Aktienreturn-Erwartung $\left(\bar{r}_{i j}^{\text {exp }}\right)$ eines Umfrageteilnehmers j für das Unternehmen i mit der folgenden Formel ermittelt:

$\bar{r}_{i j}^{\text {exp }}=\ln \left(\frac{\text { Durchschnitt }_{i j}}{100}+1\right)$

Als letztes wurde jeder Umfrageteilnehmer j gebeten, anzugeben, ob er mehr Aktien kaufen würde (Buy), seinen Aktienbestand unverändert lassen würde (Hold) oder ob er einen Teil oder alle Aktien verkaufen würde (Sell), wenn er im Besitz von Aktien des Unternehmens i wäre. Dadurch konnte eruiert werden, für welche Handlungsoption sich Umfrageteilnehmer entscheiden würden, wenn sie im Besitz von Aktien des jeweiligen fiktiven Unternehmens wären.

\subsubsection{Die Umfrageteilnehmerinnen und Umfrageteilnehmer}

Es wurden verschiedene Personenkreise kontaktiert. Die erste Gruppe waren Studentinnen und Studenten der Wirtschaftswissenschaftlichen Fakultät der Universität Basel. Die zweite Gruppe stellten institutionelle Anleger und Finanzberater dar. Es wurde bei Versicherungen, Banken und bei Vermögensverwaltern angefragt, ob sich eine oder mehrere Personen im Unternehmen dazu bereit erklären würden, den Umfragebogen auszufüllen. Der dritte Personenkreis setzte sich aus Personen aus dem Umfeld des Autors, die einer beruflichen Tätigkeit im Finanzsektor nachgehen und/oder in ihrer beruflichen Position Investitionsentscheidungen treffen, zusammen.

\subsection{Hypothesen}

Basierend auf den Studienresultaten zum Einfluss von negativen Worten in medialer Berichterstattung, zum Medienpessimismus und zum News-Tone von Tetlock et al. (2008), Tetlock (2007), und Bosman et al. (2015) wird die folgende Hypothese wie folgt formuliert:

H1: Investoren prognostizieren einen signifikant höheren (tieferen) Aktienreturn, wenn sie ein positiv (negativ) formuliertes Managementstatement lesen.

Werden in Managementstatements rechtliche/strittige Sachverhalte kommuniziert, deutet dies auf bevorstehende oder bereits existierende mit dem Gesetz in Verbindung stehende mögliche Problematiken für das Unternehmen hin. Durch rechtliche/strittige Sachverhalte generierte Eventualitäten können das Unternehmen in seiner weiteren Entwicklung vor grosse Probleme stellen. Dieser Logik folgend, wird die folgende Hypothese formuliert:

H2: Investoren prognostizieren einen signifikant tieferen (höheren) Aktienreturn, wenn sie ein rechtlich/strittig (neutral) formuliertes Managementstatement lesen.

Bildet ein Investor nach dem Lesen eines Managementstatements Erwartungen und Einschätzungen, ist neben den beschriebenen Sachverhalten entscheidend, wie sicher diese aus seiner Sicht zutreffend sind. Wird die Zutreffens-Wahrscheinlichkeit als höher eingestuft, 
wird seitens der Investoren mit extremeren Einschätzungen gerechnet. Basierend auf diesen Annahmen wird folgende Hypothese aufgestellt:

H3: Investoren prognostizieren einen signifikant tieferen (böheren) absoluten Aktienreturn, wenn sie ein unsicher (neutral) formuliertes Managementstatement lesen.

In gleicher Weise wie bei Hypothese 1 basiert die Hypothese zur Durchschnittsreturn-Prognose auf den Ergebnissen der Studien von Tetlock et al. (2008), Tetlock (2007) und Bosman et al. (2015).

H4: Investoren sind der Meinung, dass die Gesamtheit der Investoren einen signifikant höberen (tieferen) Aktienreturn prognostiziert, wenn ein positiv (negativ) formuliertes Managementstatement gelesen wird.

Um herauszufinden, ob sich die Mittelwerte der jeweiligen Schätzungen signifikant unterscheiden, werden jeweils zweiseitige t-Tests mit der Nullhypothese durchgeführt, dass sich die Mittelwerte nicht voneinander unterscheiden.

Gemäss der anfangs formulierten Definition des Begriffs Reaktionsweise sind die aus den Erwartungsanpassungen abgeleiteten Handelsentscheidungen nicht Teil der Reaktionsweise der Investoren. Deshalb werden die diesbezüglichen Antworten der Umfrageteilnehmer gesondert analysiert.

\subsection{Daten}

Insgesamt wurde der Umfragebogen 242 Mal ausgefüllt und die Umfrage war insgesamt drei Wochen geöffnet. Nach der Einspeisung wurden die Daten in einem zweiten Schritt bereinigt. Über den Verbleib im Datensample entschieden vorab formulierte Verbleibkriterien. Diese gaben bspw. eine Bearbeitungsmindestzeit vor oder wiesen auf sich widersprechende Antworten bei Umfrageteilnehmern hin. Final verblieben 136 Beobachtungen im Datensample. In einem nächsten Schritt wurden deskriptive Statistiken erstellt und Hypothesentests durchgeführt.

Bei der Charakteristikaverteilungsanalyse der drei Umfragestränge zeigte sich das Bild, dass in den drei Strängen die Charakteristika über die drei Stränge hinreichend gleichverteilt war, um mit der empirischen Analyse auf geplante Weise fortfahren zu können.

\section{Empirische Analyse}

\subsection{Die Ergebnisse zu den Aktienreturn-Erwartungen}

Die Resultate zu den Aktienreturn-Prognosen suggerieren, dass die unerfahrenen Privatanleger einen signifikant höheren Aktienreturn prognostizieren, wenn sie positiv formulierte Managementstatements lesen. Des Weiteren scheinen sie einen signifikant tieferen Aktienreturn zu prognostizieren, wenn sie negativ formulierte Managementstatements lesen. Bei der positiven und der negativen Einfärbung resultierte eine Ein-Monatsreturn-Prognose von $1.66 \%$ bzw. von $-1.37 \%$. Die Differenz von $3.03 \%$ war mit einem p-Wert von $0.0027 * *$ signifikant. Die Ein-Monatsreturn-Prognosewerte der jeweiligen Investorentypen sind in der untenstehenden Tabelle dargestellt. Es kann an dieser Stelle festgehalten werden, dass die Art der Formulierung von Managementstatements im Fall von positiver bzw. negativer Formulierung die Erwartungen und Einschätzungen der unerfahrenen Privatanleger im Sinne von Hypothese 1 beeinflusst. Bei den professionellen Anlegern und 
den erfahrenen Privatanlegern zeigte sich ein anderes Bild. Ihre Einschätzungen und Erwartungen wurden durch die Art der Formulierung im Fall von positiver bzw. negativer Formulierung nicht signifikant beeinflusst.

\begin{tabular}{|l|l|c|c|c|c|}
\cline { 2 - 6 } \multicolumn{2}{c|}{} & Mean & Median & SD & N \\
\hline \multirow{3}{*}{ Unerfahrene Privatanleger } & positiv & 0.0166 & 0.0188 & 0.0600 & 83 \\
\cline { 2 - 6 } & neutral & -0.0066 & -0.0141 & 0.0631 & 83 \\
\cline { 2 - 6 } & negativ & -0.0137 & -0.0121 & 0.0681 & 83 \\
\hline \multirow{3}{*}{ Erfahrene Privatanleger } & positiv & -0.0107 & -0.0161 & 0.0703 & 31 \\
\cline { 2 - 6 } & neutral & 0.0084 & 0.0030 & 0.0909 & 31 \\
\cline { 2 - 6 } & negativ & 0.0020 & 0.0010 & 0.0982 & 31 \\
\hline \multirow{3}{*}{\begin{tabular}{l} 
Professionelle Anleger \\
\cline { 2 - 6 }
\end{tabular}} & positiv & -0.0363 & -0.0367 & 0.0666 & 22 \\
\cline { 2 - 6 } & neutral & -0.0190 & -0.0106 & 0.0837 & 22 \\
\cline { 2 - 6 } & negativ & -0.0244 & 0.0154 & 0.0830 & 22 \\
\hline
\end{tabular}

Tabelle zu den Aktienreturn-Prognosen: In dieser Tabelle sind die deskriptiven Statistiken für die Aktienreturn-Prognosen der unerfahrenen Privatanleger, der erfahrenen Privatanleger und der professionellen Anleger abgebildet. Für alle Ausprägungen ist der Mittelwert (Mean), der Medianwert (Median), die Standardabweichung (SD) und die Anzahl der Beobachtungen (N) aufgeführt. Die Werte wurden auf vier Nachkommastellen gerundet.

Entgegen des erwarteten Zusammenhangs prognostizierte keiner der drei Investorentypen einen signifikant tieferen (höheren) Aktienreturn im Sinne von Hypothese 2, wenn rechtlich/strittig (neutral) formulierte Managementstatements gelesen wurden. Die Ergebnisse lassen den Rückschluss zu, dass in diesem Fall die Art der Formulierung keine signifikante Wirkung entfaltet.

Die Ergebnisse zu Hypothese 3 deuten entgegen der formulierten Vermutung stark darauf hin, dass Investoren nicht dazu neigen, einen tieferen (höheren) absoluten Aktienreturn zu prognostizieren, wenn sie ein unsicher (neutral) formuliertes Managementstatement lesen. In der vorliegenden Untersuchung konnte nicht festgestellt werden, dass bei Ungenauigkeit weniger extreme Erwartungen und Einschätzungen gebildet werden.

Die im Rahmen dieser Untersuchung generierten Daten zu den Volatilitätsprognosen deuten darauf hin, dass alle Investorentypen ihre Volatilitätsprognose losgelöst von der Art der Formulierung erstellen. Die Erwartungen und Einschätzungen zur Volatilität variieren somit nicht mit der Art der Formulierung, was in der Konsequenz impliziert, dass die Art der Formulierung in den Augen der Investoren keine relevanten Informationen enthält.

\subsection{Die Ergebnisse zu den Durchschnittsreturn-Erwartungen}

Konform mit dem erwarteten Zusammenhang bei Hypothese 4 deuten die Ergebnisse zu den Durchschnittsreturn-Prognosen bei den unerfahrenen Privatanlegern darauf hin, dass sie der Meinung sind, dass die Gesamtheit der Investoren einen signifikant höheren (tieferen) Aktienreturn prognostiziert, wenn positiv (negativ) formulierte Managementstatements gelesen werden. Bei der positiven und der negativen Einfärbung resultierten EinMonatsdurchschnittsreturn-Prognosen von 1.16\% und $-1.73 \%$. Die Differenz von 2.89\% 
war signifikant. Vergleicht man diese Werte mit den Werten der unerfahrenen Privatanleger zu der positiven und der negativen Einfärbung bei den Aktienreturn-Prognosen (Positiv: $1.66 \%$, Negativ: $-1.37 \%$, Differenz: $3.03 \%$ ), fällt auf, dass die beiden signifikanten Differenzen einen sehr ähnlichen Wert aufweisen. Die Ergebnisse zu den Durchschnittsreturn-Prognosen der unerfahrenen Privatanleger suggerieren, dass die Art der Formulierung von Managementstatements im Fall von positiver bzw. negativer Formulierung die Erwartungen und Einschätzungen der unerfahrenen Privatanleger im Sinne von Hypothese 4 beeinflusst. Bei den professionellen Anlegern und den erfahrenen Privatanlegern war es erneut so, dass ihre Einschätzungen und Erwartungen nicht signifikant durch die Art der Formulierung im Fall von positiver bzw. negativer Formulierung beeinflusst wurden.

Bei den unerfahrenen Privatanlegern wird somit auf der Individuum-Ebene erwartet, dass die Gesamtheit der Investoren ihre Erwartungen zu den Aktienreturn-Prognosen bezüglich positiv bzw. negativ formulierter Managementstatements auf die gleiche Weise bilden wie sie. Gleich wie zuvor bei den unerfahrenen Privatanlegern wird bei den professionellen Anlegern und den erfahrenen Privatanlegern auf der Individuum-Ebene erwartet, dass die Gesamtheit der Investoren ihre Erwartungen zu den Aktienreturn-Prognosen bezüglich des Tone in Managementstatements auf die gleiche Weise bilden wie sie. Während die professionellen Anleger und die erfahrenen Privatanleger gemäss den Ergebnissen davon ausgehen, dass der Tone in Managementstatements sowohl auf sie als auch auf die unerfahrenen Privatanleger keine signifikante Wirkung erzielt, gehen die unerfahrenen Privatanleger gemäss den Ergebnissen davon aus, dass der Tone in Managementstatements sowohl auf sie als auch auf die professionellen Anleger und die erfahrenen Privatanleger eine signifikante Wirkung erzielt. Dies bedeutet, dass in dieser Hinsicht die unerfahrenen Privatanleger bezüglich der Erwartungen der professionellen Anleger bzw. der erfahrenen Privatanleger einer Fehleinschätzung unterliegen, et vice versa.

\subsection{Die Ergebnisse zu den gewählten Handlungsoptionen}

Bei den gewählten Handlungsoptionen wurde die Tendenz vermutet, dass Investoren eher zu Buy (Sell) neigen, wenn sie positiv (negativ) formulierte Managementstatements lesen. Diese Tendenz erhält von den vorliegenden Resultaten dieser Untersuchung wenig bis keine Unterstützung. Die Resultate deuten darauf hin, dass die Art der Formulierung im Fall von positiver bzw. negativer Einfärbung keine Wirkung auf die erfahrenen Privatanleger und eine geringe Wirkung auf die unerfahrenen Privatanleger und die professionellen Anleger entfaltet.

Die Ergebnisse zu den gewählten Handlungsoptionen deuten jedoch darauf hin, dass Investoren eher zu Sell (Buy) neigen, wenn sie rechtlich/strittig (neutral) formulierte Managementstatements lesen. Insbesondere bei den professionellen Anlegern und den erfahrenen Privatanlegern schien diese Neigung vorzuliegen. Bei den unerfahrenen Privatanlegern war eine geringe Neigung in diese Richtung auszumachen. Die Resultate suggerieren, dass die Art der Formulierung im Fall von rechtlicher/strittiger Formulierung bei den gewählten Handlungsoptionen eine Wirkung entfaltet.

Die verschiedenen Investorentypen neigten in der vorliegenden Untersuchung nicht oder nur geringfügig dazu, sich für Hold (Buy/Sell) auszusprechen, wenn unsicher (neutral) formulierte Managementstatements gelesen wurden. Die Ergebnisse deuten darauf hin, dass die Art der Formulierung im Fall der unsicheren Formulierung keine oder bloss eine geringe Wirkung auf die gewählten Handlungsoptionen entfaltet. 


\section{Konklusion}

In dieser Arbeit wurde eine neuartige Informationsquelle für die Analyse textbasierten Sentiments genutzt. Durch die Implementierung einer neutralen Referenz konnten nicht bloss die Erwartungen bei positiver und bei negativer Einfärbung verglichen werden, sondern darüber hinaus konnten die Erwartungen bei positiver bzw. negativer Einfärbung zusätzlich mit den Erwartungen bei einer neutralen Referenz verglichen werden. Zusätzlich ermöglichte die Implementierung einer neutralen Referenz die Untersuchung, ob die Erwartungen bei unsicherer bzw. rechtlicher/strittiger Einfärbung von den Erwartungen bei einer neutralen Referenz divergieren.

In Anbetracht dessen, dass die Erwartungen und Einschätzungen der unerfahrenen Privatanleger bezüglich der Aktienreturn-Prognosen und der Durchschnittsreturn-Prognosen mit der Art der Formulierung variieren, lässt sich schlussfolgern, dass die Art der Formulierung in den Augen dieses Investorentyps, bewusst und/oder unbewusst wirkend, relevante Informationen enthält. Es kann konstatiert werden, dass die Ergebnisse der vorliegenden Untersuchung eine kausale Beziehung von Investoren-Sentiment und textbasiertem Sentiment aufzeigen.

Aus einer praktischen Sicht würde dies bedeuten, dass seitens des Managements systematisch auf die Erwartungen und Einschätzungen unerfahrener Privatanleger Einfluss genommen werden kann. Angenommen, ein Managementteam eines Unternehmens befindet sich in einer Lage, in welcher es aus seiner Sicht sinnvoll/wünschenswert ist, die Erwartungen und Einschätzungen eines Teils der Investoren in eine gewünschte Richtung zu beeinflussen, ist dies gemäss den Ergebnissen der vorliegenden Arbeit durch eine Manipulation des Tone temporär möglich. Divergieren aus der Sicht des Managements zu einem Zeitpunkt die tatsächliche aktuelle Unternehmenslage und die durch die Finanzkennzahlen wiedergegebene aktuelle Unternehmenslage, wäre es demnach möglich, durch eine Manipulation des Tone in einem oder mehreren Managementstatements die aktuelle Unternehmenslage zu beschönigen/beeinträchtigen, wodurch die Deckungsgleichheit der Aussenwahrnehmung und der Sicht des Managements erreicht werden kann.

In dieser Arbeit stellt die Gesamtanzahl der Daten eine bedeutende Restriktion dar. Des Weiteren hängt die Eignung der Daten in hohem Masse von den erstellten fiktiven Managementstatements, den gestellten Fragen und bezüglich der Managementstatements insbesondere davon ab, ob die linguistischen Einfärbungen die Treatment-Statements wie beabsichtigt modifizierten. Obwohl bei den Treatment-Statements die Referenzstatements auf eine objektiv begründbare Weise eingefärbt wurden, lässt sich letztlich nicht mit Sicherheit feststellen, ob alle Referenzstatements wie beabsichtigt eingefärbt werden konnten.

Die Verbindung und die kausalen Beziehungen von Investoren-Sentiment und textbasiertem Sentiment sind hochgradig komplex. Es ist wünschenswert, dass zukünftige Forschung in diesem Bereich intensiviert wird. Wie textbasiertes Sentiment auf verschiedene Investorentypen wirkt und inwiefern textbasiertes Sentiment auf das Investoren-Sentiment der verschiedenen Investorentypen wirkt, bedarf aus Sicht des Autors weiterer Forschung. Es ist denkbar, dass insbesondere der innovative Ansatz des Machine Learning die Erforschung der kausalen Beziehungen von Investoren-Sentiment und textbasiertem Sentiment vorantreiben wird. 


\section{Literaturverzeichnis}

Baker, M., \& Wurgler, J. (2006). Investor Sentiment and the Cross-Section of Stock Returns. The Journal of Finance, 61(4), 1645-1680.

Baker, M., \& Wurgler, J. (2007). Investor Sentiment in the Stock Market. Journal of Economic Perspectives, 21(2), 129-151.

Black, F. (1986). Noise. The Journal of Finance, 41(3), 528-543.

Bosman, R., Kräussl, R., \& Mirgorodskaya, E. (2015). The “Tone Effect” of News on Investor Beliefs: An Experimental Approach. CFS Working Paper Series.

De Long, J. B., Shleifer, A., Summers, L. H., \& Waldmann, R. J. (1990). Noise trader risk in financial markets. Journal of Political Economy, 98(4), 703-738.

Kearney, C., \& Liu, S. (2014). Textual sentiment in finance: A survey of methods and models. International Review of Financial Analysis, 33, 171-185.

Li, F. (2010). The information content of forward- looking statements in corporate filings - A naïve bayesian machine learning approach. Journal of Accounting Research, 48(5), 1049-1102.

Loughran, T., \& Mcdonald, B. (2011). When is a liability not a liability? Textual analysis, dictionaries, and 10-Ks. The Journal of Finance, 66(1), 35-65.

Shleifer, A., \& Vishny, R. W. (1997). The Limits of Arbitrage. The Journal of Finance, 52(1), 35-55.

Stock, J. H., \& Watson, M. W. (2011). Introduction to Econometrics, 3rd ed. Introduction to Econometrics, 3 rd ed.

Tetlock, P. C. (2007). Giving Content to Investor Sentiment: The Role of Media in the Stock Market. The Journal of Finance, 62(3), 1139-1168.

Tetlock, P. C., Saar-Tsechansky, M., \& Macskassy, S. (2008). More Than Words: Quantifying Language to Measure Firms' Fundamentals. The Journal of Finance, 63(3), 1437-1467.

Joachim Lang, M.Sc., war Masterstudent an der Wirtschaftswissenschaftlichen Fakultät der Universität Basel, E-Mail: joachim.johannes.lang@gmail.com

Anschrift: Universität Basel, Wirtschaftswissenschaftliche Fakultät, Peter Merian-Weg 6, 4052 Basel 